\title{
Continuous endometrial volumetric analysis for endometrial receptivity assessment on assisted reproductive technology cycles
}

\author{
Renato Silva Martins ${ }^{1,2^{*}}$ (D) António Helio Oliani ${ }^{1}$, Denise Vaz Oliani ${ }^{1}$ and José Martinez de Oliveira ${ }^{1,2}$
}

\begin{abstract}
Background: Human implantation is a complex process requiring synchrony between a healthy embryo and a functionally competent or receptive endometrium. In order to assess endometrial receptivity in Assisted Reproductive Technology (ART) cycles serial evaluation of endometrial volumetric analysis may have a predictive value on a positive outcome.

Methods: Serial 3D transvaginal ultrasound performed in women on ART cycle to evaluate embryo implantation predictors. Prospective case control study of 169 subjects were assessed. Endometrial pattern, thickness, volume and adjusted endometrial volume (ratio between endometrial volume and uterine volume) was performed to all subjects on a continuous process from baseline, during controlled ovarian stimulation, trigger day with human chorionic gonadotropin hormone (hCG) and at embryo transfer day.

Results: Demographics and ART procedures and scores, was similar between the two groups. Endometrial morphology also showed no difference between the two groups. Endometrial volume and adjusted endometrial volume was significantly higher in the positive group as soon as day 6 of ovarian controlled stimulation.

Conclusions: Serial 3D endometrial volume and adjusted endometrial volumes provides a predicting clinical tool enhancing elective embryo transfers in fresh ART cycle. Thus providing a non-invasive continuous technique for endometrial receptivity assessment that reflects endometrial changes during ART procedures.
\end{abstract}

Keywords: Endometrial receptivity, Assisted reproductive technology, Endometrial volume, Adjusted endometrial volume, Embryo implantation

\section{Background}

Successful assisted reproductive technology cycles outcome depends on the intricate interplay between embryo quality and endometrial receptivity. Endometrium is a dynamic tissue that grows, differentiates and suffers regression throughout the menstrual cycle in response to hormonal regulation to prepare the uterus for embryo

\footnotetext{
* Correspondence: renato.alessandre@gmail.com

${ }^{1}$ Centro Hospitalar Universitário Cova da Beira EPE, Quinta do Alvito, 6200 503 Covilha, Portugal

${ }^{2}$ Centro Investigação Ciências da Saúde - Faculdade Ciências da Saúde, Universidade da Beira Interior, Alameda Infante D, Henrique, 6200506 Covilha, Portugal
}

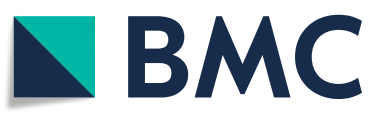

(c) The Author(s). 2020 Open Access This article is licensed under a Creative Commons Attribution 4.0 International License, which permits use, sharing, adaptation, distribution and reproduction in any medium or format, as long as you give appropriate credit to the original author(s) and the source, provide a link to the Creative Commons licence, and indicate if changes were made. The images or other third party material in this article are included in the article's Creative Commons licence, unless indicated otherwise in a credit line to the material. If material is not included in the article's Creative Commons licence and your intended use is not permitted by statutory regulation or exceeds the permitted use, you will need to obtain permission directly from the copyright holder. To view a copy of this licence, visit http://creativecommons.org/licenses/by/4.0/ The Creative Commons Public Domain Dedication waiver (http://creativecommons.org/publicdomain/zero/1.0/) applies to the data made available in this article, unless otherwise stated in a credit line to the data.

implantation [1]. Endometrium is a highly dynamic tissue undergoing physiological changes in response to ovarian steroid hormones. It has been proven that the supraphysiological hormonal levels as in the ART cycle has a harmful effect on endometrial receptivity. Endometrial characteristics compatible with a successful pregnancy have proven to be difficult to be properly assessed. Adequate endometrial development seems to be important for implantation given that previous studies have shown an association between abnormal glandular or vascular development and defective placentation disorders. The window of implantation (WOI) is defined as a short period of 
time while the endometrium is receptive to the embryo $[2,3]$.

Diagnosis of endometrial receptivity (ER) has posed a challenge and so far, most available tests have been subjective and lack accuracy and a predictive value [4].

The use of transcriptomic signature of the WOI by microarray technology is possible, however it demands an endometrial biopsy [5]. This requires an invasive procedure and it has an associated cost. Also, in women with irregular cycles it may not prove to be cost-efficient [6].

Ultrasound can asses changes in the endometrium during stimulated cycles. It also has minimal interobserver and intra-observer variability. Monitoring of both the endometrial and ovarian response to ovarian stimulation on ART cycles with transvaginal ultrasound has become an important predictor of the success of ART. Published studies have conflicting results on this subject, but the common feature in all, is the lack of continuity on endometrial assessment [7]. The use of high-resolution transvaginal probes makes it possible to follow endometrium changes throughout the cycle [8]. From a clinical point of view some objective parameters must be obtained in order to ascertain the likelihood of an ongoing pregnancy in ART cycles, preferably in a non-invasive and cost-efficient way [9]. Some published work has recently proven a pattern of hemodynamic changes in utero-ovarian arteries during ART cycles with predictive value on endometrial receptivity [10]. Hou et al. have also confirmed the possibility of noninvasive prediction of success in ART cycles, with serial assessments of the echogenicity pattern transformation, after human recombinant gonadrotropin hormone [11]. The aim of this prospective study is to further evaluate the capability of serial and continuous evaluation of biophysical markers as a non-invasive procedure to determine endometrial receptivity $[12,13]$.

\section{Methods}

Prospective cohort study of 169 women in ART cycles. All infertile couples submitted to ART treatment at our institution were included from January 2017 to December 2018 (2 Year period). Canceled treatments prior to oocyte pickup; cycles with missing or erroneous data; and cycles with elective single embryo transfer were excluded. (Fig. 1.)

The primary data source for this study was the local databases routinely used in the participating centre in ongoing treatments. The data output was anonymized in the extraction for statistical treatment purposes. All data collected and written informed consent was obtained according to the Ethics Committee of our Institution.

Subjects with double viable good grade embryo transfer on day 3 of embryo development were included. All subjects have been in a short protocol regimen with antagonist for ovarian controlled stimulation using gonadotropins. All subjects were submitted to recombinant human chorionic gonadotropin hormone (rhCG) for induction of ovulation $36 \mathrm{~h}$ prior to oocyte pick up.

Ultasonographic protocol for serial ultrasound analysis (endometrial morphology, endometrial thickness, endometrial volume and uterine volume) was performed.

During ovarian controlled stimulation serial ultrasound exams were performed, using 3D transvaginal probe.

Ultrasonographic markers were obtained in all evaluations (Basal moment - day 2 or 3 of women menstrual cycle and prior to onset of ovarian controlled stimulation; at day 6, day 8 and day 10 after initiating ovarian controlled stimulation; at Trigger day with recombinant human gonadochorionic hormone; and at embryo transfer day).

Endometrial morphology was based on the two grade system by Sher et al. [14]: non-multilayered homogeneous hyperechogenic or iso-echogenic endometrium compared with the myometrium and multilayered tripleline pattern, 'halo pattern' with an outer peripheral layer of denser echogenicity and a central sonolucent area.

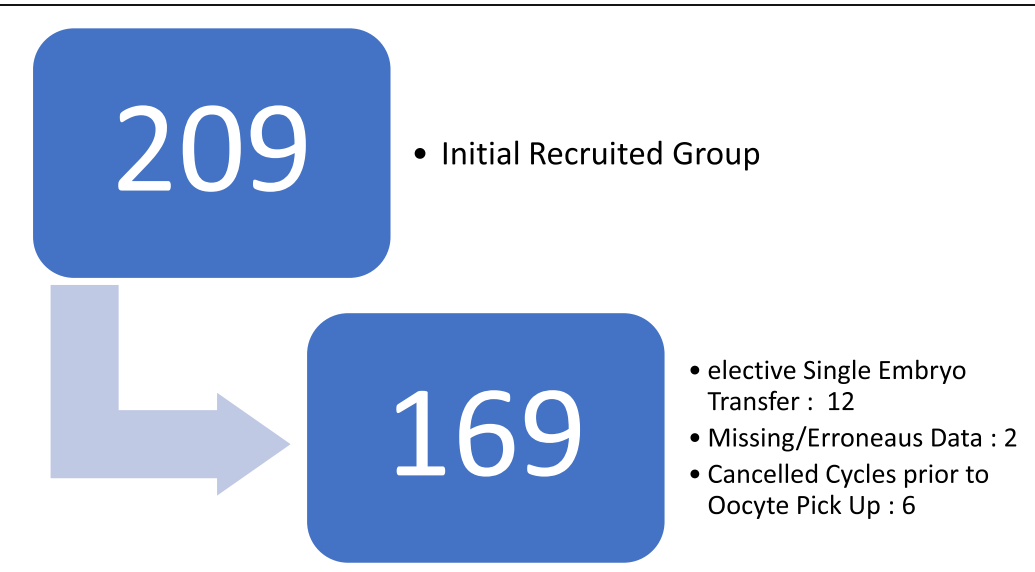

Fig. 1 Study Population and Exclusion Criteria 
Endometrial thinckness was obtained in milimeters $(\mathrm{mm})$ on the long axis or sagittal plane, with the entirely of the endometrial lining through and endocervical canal in view. The measurement was taken of the thickest echogenic area from one basal endometrial interface across the endometrial canal to the other basal surface.

Endometrial volume calculation by $3 \mathrm{D}$ ultrasound presented as voxels and geometric information of surfaces in a $3 \mathrm{D}$ dataset. The results obtained are then converted to mililitres. Adjusted Endometrial volume was also obtained as a ratio between endometrial volume calculated on 3D analysis and uterine volume based on 3D volumetric acquisitions which then generated an estimated uterine volume (also in mililiters). Adjusted endometrial volume deflects the potential difference in uterine volume from each single individual.

At day 12 after successful embryo transfer, human gonadochorionic sub-unit B serum levels were obtained, and groups were set: positive results (for values over 5 International Units - IU) and negative results (for values under $5 \mathrm{IU}$ ). For the aim of our study the positive cases were afterwards assessed and classified with positive clinical pregnancy by the evidence of at least one viable foetus by ultrasound performed 2 weeks after the positive biochemical result.

All data collected was analysed between these two set groups and compared.

Data was analysed in Excel 2019 (Microsoft Corp, Redmond, WA) and IBM SPSS statistics v25 (IBM Corp. Armonk, NY). Continuous variables were analysed with Levene's test (equality of variances) and visual assessment of the histogram (normality).
For analysis of parametric continuous variables, a tstudent test for independent samples was used. Chisquare and Fisher's exact tests were used to analyse associations between categorical variables. Endometrial thickness, endometrial volume and adjusted endometrial volume were analysed using analysis of variance for repeated measurement data.

Value of $p<.05$ was considered statistically significant.

The authors do not report any conflict of interest.

The study protocol has been approved by the Ethics Committee of our Institution (CHCB 22/2017), in accordance with the relevant guidelines and regulations. This study has been conducted in accordance with legal and regulatory requirements, as well as follow generally accepted research practices described in International Conference Harmonisation ( $\mathrm{ICH}$ ) guidelines, Good Clinical Practices (GCP) and the Declaration of Helsinki.

\section{Results}

Clear morphology and volume of the endometrium was obtained in all 169 cycles using 3D transvaginal ultrasound in continuous serial observations. Demographics characteristics and ART parameters are shown in Table 1.

Women were divided into two groups depending on the value of hCG at Day 12 after embryo transfer and ultrasound confirmation of clinical pregnancy: 123 on the negative group (72.8\%) and 46 on the positive group (27.2\%).

There were no statistical difference between the two set groups in terms of demographics and ART parameters: mean age of female partner or mean age of male

Table 1 Demographics and ART parameters between two Groups

\begin{tabular}{|c|c|c|c|}
\hline & $\begin{array}{l}\text { Negative Group } \\
N=123(72.8 \%)\end{array}$ & $\begin{array}{l}\text { Positive Group } \\
N=46(27.2 \%)\end{array}$ & $\begin{array}{l}\mathrm{t} \text {-Test } \\
p \text { value }\end{array}$ \\
\hline Female Age (in years) & $34.94 \pm 4.03(19-39)$ & $34.28 \pm 3.35(25-39)$ & 0.290 \\
\hline Male Age (in years) & $36.14 \pm 4.76(22-46)$ & $37.19 \pm 5.91(29-62)$ & 0.832 \\
\hline Time of Infertility (in months) & $54.46 \pm 33.82(12-204)$ & $60.22 \pm 38.49(14-192)$ & 0.375 \\
\hline \multicolumn{4}{|l|}{ Type of Infertility: } \\
\hline • Primary & $95 / 123(77.2 \%)$ & $38 / 46(82.6 \%)$ & \multirow[t]{2}{*}{0.297} \\
\hline - Secondary & $28 / 123(22.8 \%)$ & $8 / 46(17.4 \%)$ & \\
\hline Antimullerian hormone $(\mathrm{pg} / \mathrm{mL})$ & $2.45 \pm 2.45(0.09-16.65)$ & $2.62 \pm 2.46(0.04-13.56)$ & 0.679 \\
\hline Antral follicle count & $8.43 \pm 5.07(2-40)$ & $8.63 \pm 3.74(2-20)$ & 0.801 \\
\hline Total dose of gonadotropins (in International Units) & $2500.81 \pm 812.19(300-4500)$ & $2508.15 \pm 757.91(450-4500)$ & 0.956 \\
\hline Progesterone levels at Trigger day (ng/mL) & $0.88 \pm 0.44(0.01-2.20)$ & $0.78 \pm 0.47(0.01-2.10)$ & 0.188 \\
\hline Number of collected Oocytes & $8.25 \pm 5.14(2-22)$ & $10.50 \pm 5.20(2-23)$ & 0.140 \\
\hline Metaphase II Oocytes & $6.57 \pm 4.22(2-17)$ & $7.06 \pm 4.77(2-21)$ & 0.150 \\
\hline Number of day 3 embryos & $3.18 \pm 2.40(2-12)$ & $3.84 \pm 2.65(2-12)$ & 0.120 \\
\hline Number of blastocyst for vitrification & $0.65 \pm 1.51(0-6)$ & $0.86 \pm 1.71(0-9)$ & 0.200 \\
\hline
\end{tabular}

(Positive Group, $N=46$ and Negative Group, $N=123$ )

Descriptive statistics between two Groups. Mean values with standard deviation (SD) 
partner, duration and type of infertility, total drug dose used for ovarian stimulation, overall median number of harvested oocytes per cycle defined as the total number of oocytes harvested during oocyte pick up procedure, rate of collected metaphase II (MII) oocytes. Also, the mean number of cleaved embryos at day 3 of embryo development, and mean number of blastocysts for cryopreservation showed no significant statistical difference between the two set groups.

Endometrial morphology showed no statistically significant difference between the two set groups.

Endometrial thickness on a single 2D sagittal profile showed no statistical difference at baseline and at Day 6 after ovarian controlled stimulation. Statistically significant difference was only met at a later phase of ovarian controlled stimulation - at day 8 and on following evaluations.

Uterine Volume was comparable between the two Groups with no statistical difference between the two. (Table 2).

Endometrial Volume and Adjusted Endometrial Volume showed statistical difference from Day 6 after Ovarian controlled stimulation (Table 3 and Figs. 2 and 3). Consistently higher values were seen, for both ultrasonographic markers on the positive group. In terms of endometrial volume, the positive group had statistically significant higher values in all observations except at the basal moment prior to ovarian controlled stimulation $(2.77 \pm 0.63$ vs $2.52 \pm$ 0.71 with $p$ value of 0.54 ). Similar findings were noted on adjusted endometrial volume (Table 3).

By comparing the difference between two consecutive measurements (endometrial growth rate) for endometrial and adjusted endometrial volumes, and the overall difference between the final value and the initial basal measurement we were able to note with statistical difference that values were higher on the positive group on initial phases of endometrial development and in the overall assessment (Table 4 and Fig. 4).

In terms of endometrial volume assessment, a cut off $\geq 5 \mathrm{~mL}$ in the prediction of endometrial receptivity was used with good sensitivity (85\%) and low specificity (69\%) in a group application; in individual setting it had a good predictive negative value $(90.1 \%)$ and low predictive positive value (81.1\%), with a diagnostic accuracy of $75 \%$.

The degree of agreement was assessed by calculation of Kappa ( $\kappa$ ) statistics. $\kappa$ is a statistical parameter of agreement that does not require any assumption of the correct diagnosis, expressed through a coefficient ranging between -1.0 and +1.0 . Perfect agreement corresponds to a coefficient of 1.0, a value of zero indicates agreement no better than that expected by chance, and negative values indicate agreement worse than that expected by chance. There is no absolute way to interpret the values between 0 and 1 . As a guideline Landis and Koch indicated that values of $<0.20$ suggest poor agreement, 0.21-0.40 fair agreement, $0.41-0.60$ moderate agreement, $0.61-0.80$ good agreement and $>0.81$ excellent agreement. In this study the intra-observer reliability was 0.96 .

In addition, because all measurements were performed by the same operator in this study there was no interobserver variability.

\section{Discussion}

In this study we aimed to assess endometrial evolution in order to ascertain a plausible predictive non-invasive

Table 2 Ultrasound parameters between two Groups - Endometrial morphology and Endometrial thickness at baseline, at day 6, 8 and 10 after controlled ovarian stimulation, at trigger day and at embryo transfer day

\begin{tabular}{|c|c|c|c|c|}
\hline & & $\begin{array}{l}\text { Negative Group } \\
N=123(72.8 \%)\end{array}$ & $\begin{array}{l}\text { Positive Group } \\
N=46(27.2 \%)\end{array}$ & $p$ Value \\
\hline \multirow[t]{2}{*}{ Basal } & Endometrial Morphology (ML/NM) & $0 \% / 100 \%$ & $0 \% / 100 \%$ & NS \\
\hline & Endometrial Thickness (in mm) & $4.32 \pm 0,72$ & $4.22 \pm 0,51$ & 0.387 \\
\hline \multirow[t]{2}{*}{ Day 6 after Controlled Ovarian Stimulation } & Endometrial Morphology (ML/NM) & $78.9 \% / 21.1 \%$ & $93.5 \% / 6.5 \%$ & 0.15 \\
\hline & Endometrial Thickness (in mm) & $6.32 \pm 0.96$ & $6.28 \pm 0.75$ & 0.827 \\
\hline \multirow[t]{2}{*}{ Day 8 after Controlled Ovarian Stimulation } & Endometrial Morphology (ML/NM) & $100 \% / 0 \%$ & $100 \% / 0 \%$ & NS \\
\hline & Endometrial Thickness (in mm) & $7.47 \pm 0.80$ & $7.96 \pm 0.79$ & 0.01 \\
\hline \multirow[t]{2}{*}{ Day 10 after Controlled Ovarian Stimulation } & Endometrial Morphology (ML/NM) & $100 \% / 0 \%$ & $100 \% / 0 \%$ & NS \\
\hline & Endometrial Thickness (in mm) & $8.01 \pm 1.04$ & $8.61 \pm 0.98$ & 0.01 \\
\hline \multirow[t]{2}{*}{ Trigger Day with rhCG } & Endometrial Morphology (ML/NM) & $100 \% / 0 \%$ & $100 \% / 0 \%$ & NS \\
\hline & Endometrial Thickness (in mm) & $8.53 \pm 1.32$ & $9.59 \pm 1.44$ & 0.001 \\
\hline \multirow[t]{2}{*}{ Embryo Transfer Day } & Endometrial Morphology (ML/NM) & $4.1 \% / 95.9 \%$ & $4.3 \% / 95.7 \%$ & 0.613 \\
\hline & Endometrial Thickness (in mm) & $9.06 \pm 1.30$ & $10.15 \pm 1.35$ & 0.001 \\
\hline
\end{tabular}

Ratios in percentages (\%) and mean values with standard deviation (SD). NM Non multi-layered endometrium; ML Multi-layered endometrium; rhCG recombinant human chorionic gonadotropin; NS No statistical analysis performed 
Table 3 Ultrasound parameters between two groups - Endometrial volume and adjusted endometrial volume at baseline, at day 6, 8 and 10 after controlled ovarian stimulation, at trigger day and at embryo transfer day

\begin{tabular}{|c|c|c|c|c|}
\hline & & $\begin{array}{l}\text { Negative Group } \\
N=123(72.8 \%)\end{array}$ & $\begin{array}{l}\text { Positive Group } \\
N=46(27.2 \%)\end{array}$ & $\begin{array}{l}\mathrm{t} \text {-Test } \\
p \text { Value }\end{array}$ \\
\hline \multirow[t]{2}{*}{ Basal } & Endometrial Volume (in mm3) & $2.52 \pm 0.71$ & $2.77 \pm 0.63$ & 0.54 \\
\hline & Adjusted Endometrial Volume & $4.60 \pm 1.42$ & $5.51 \pm 1.28$ & 0.21 \\
\hline \multirow[t]{2}{*}{ Day 6 after Controlled Ovarian Stimulation } & Endometrial Volume (in mm3) & $3.08 \pm 0.66$ & $3.33 \pm 0.57$ & 0.024 \\
\hline & Adjusted Endometrial Volume & $5.63 \pm 1.50$ & $6.67 \pm 1.38$ & 0.001 \\
\hline \multirow[t]{2}{*}{ Day 8 after Controlled Ovarian Stimulation } & Endometrial Volume (in mm3) & $3.90 \pm 0.94$ & $4.40 \pm 0.71$ & 0.002 \\
\hline & Adjusted Endometrial Volume & $7.28 \pm 2.67$ & $8.98 \pm 2.47$ & 0.001 \\
\hline \multirow[t]{2}{*}{ Day 10 after Controlled Ovarian Stimulation } & Endometrial Volume (in mm3) & $4.12 \pm 1.01$ & $4.91 \pm 0.82$ & 0.001 \\
\hline & Adjusted Endometrial Volume & $7.60 \pm 2.54$ & $9.99 \pm 2.61$ & 0.001 \\
\hline \multirow[t]{2}{*}{ Trigger Day with rhCG } & Endometrial Volume (in mm3) & $4.52 \pm 1.00$ & $5.33 \pm 0.76$ & 0.001 \\
\hline & Adjusted Endometrial Volume & $8.30 \pm 2.52$ & $10.76 \pm 2.62$ & 0.001 \\
\hline \multirow[t]{2}{*}{ Embryo Transfer Day } & Endometrial Volume (in mm3) & $4.84 \pm 1.01$ & $5.59 \pm 0.77$ & 0.001 \\
\hline & Adjusted Endometrial Volume & $8.32 \pm 2.58$ & $10.83 \pm 2.73$ & 0.001 \\
\hline
\end{tabular}

Ratios in percentages (\%) and mean values with standard deviation (SD). rhCG recombinant human chorionic gonadotropin

diagnostic tool for clinicians to better understand endometrium changes.

The pregnancy potential of good quality embryos is still not high on ART cycles, even with the progress in the programs of ovarian stimulation, ART technique and embryo development and culture. Implantation is still strongly reliable on the cross talk between a healthy good quality embryo and the receptive endometrium.

Although several parameters have been used to assess the pregnancy rate in ART cycles, there is still some controversy about its efficacy, and underlying mechanisms in endometrial receptivity [15-18]. Vaginal 3D ultrasound is a non-invasive and an inexpensive tool at clinician's disposal [19]. The process of endometrial transformation from proliferative phase to secretory phase under the steroids hormonal influence, called endometrial decidualization is a set goal for optimal implantation. The cyclic changes of endometrium are regulated by ovarian hormones and its receptors, and endometrial luteal phase development may alter in ART cycles due to supraphysiological hormone levels.

Contradictory findings is often reported in single analysis of endometrial pattern at trigger day.

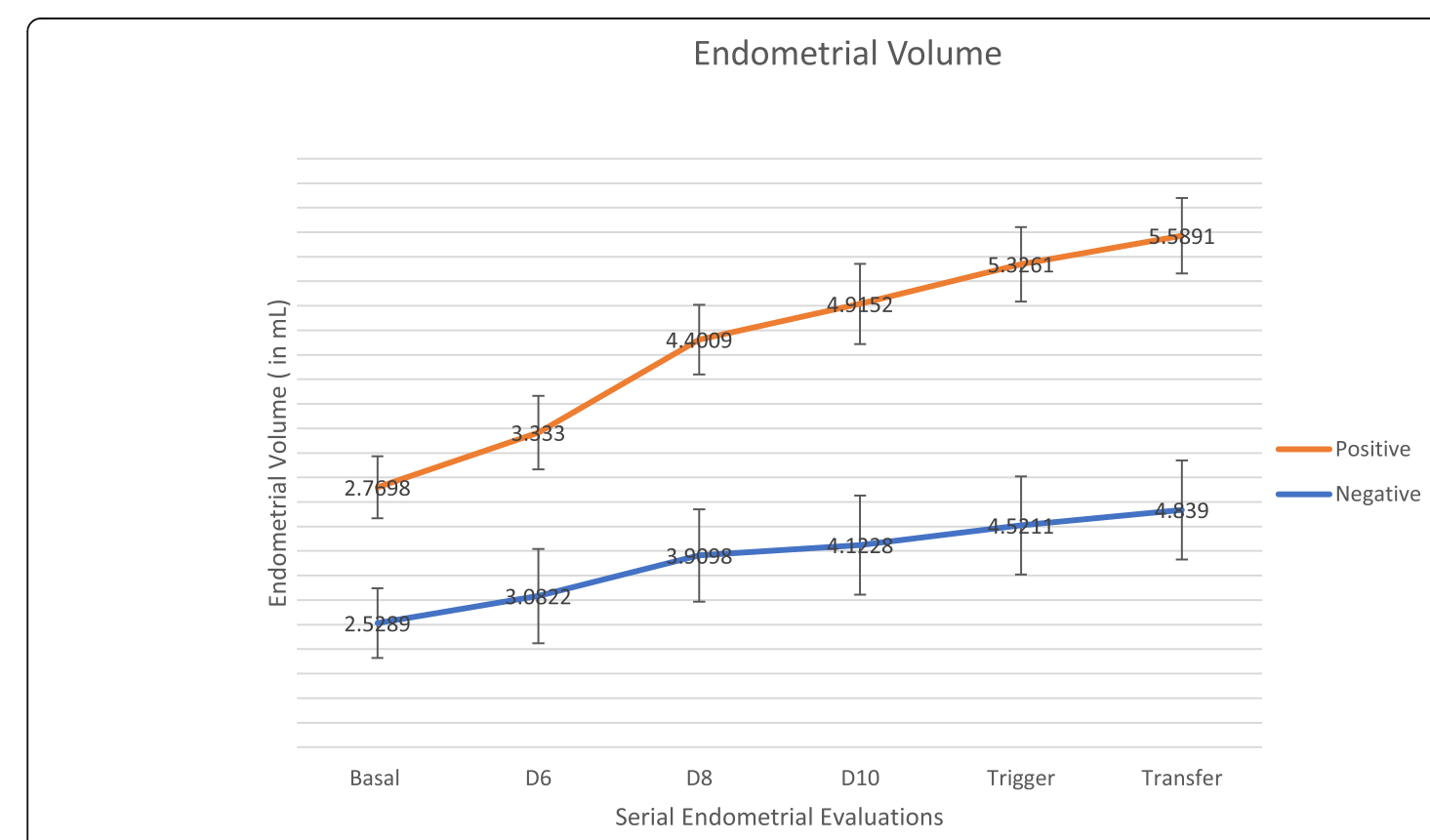

Fig. 2 Continuous endometrial volume analysis (Mean values with Standard Deviation) 


\section{Ajusted Endometrial Volume}

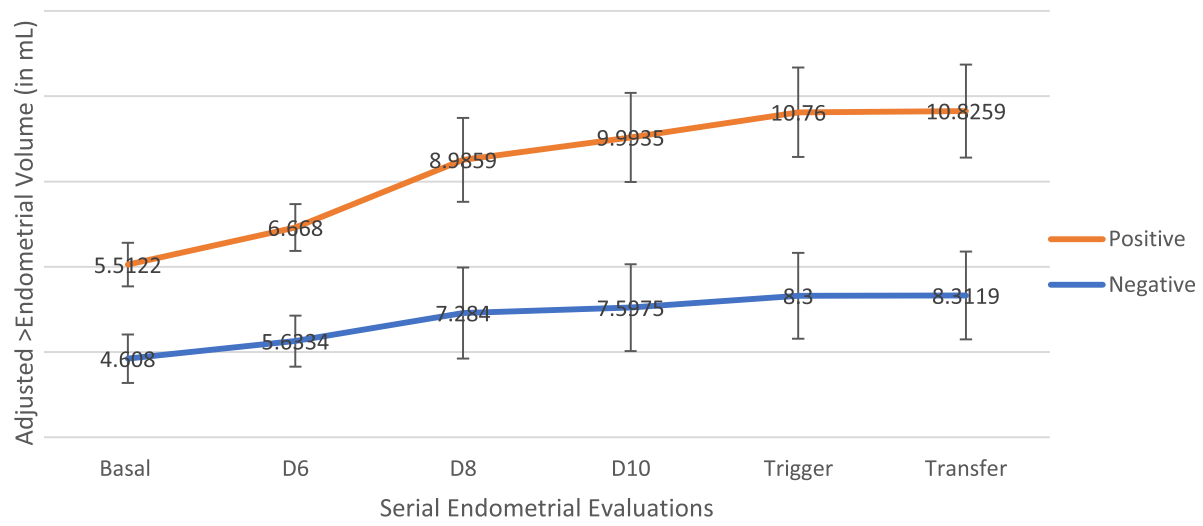

Fig. 3 Continuous adjusted endometrial volume analysis. (Mean values with Standard Deviation)

Recent studies (Silva Martins, R. et al.) have proven that perhaps serial evaluations provide better understanding rather than a single scoop at a pre-determined phase of the process. It has been proven that in terms of angiogenesis that there is a certain pattern of evolution. This relates to what one should expect from a transforming living tissue and its natural adaptations on the complex binding process of implantation.

The main purpose of this study was to further evaluate potential ultrasonographic markers that might be evaluated in the continuous changes that endometrium goes

Table 4 Endometrial and Adjusted Endometrial volume Growth rate (difference between two consecutive continuous evaluations, and Overall difference between final and first evaluation)

\begin{tabular}{lllllll}
\hline \multicolumn{7}{l}{ Endometrial Volume Growth Rate } \\
\hline & GR1 & GR2 & GR3 & GR4 & GR5 & Overall GR \\
\hline Negative EV & 0,5533 & 0,8275 & 0,4357 & 0,3626 & 0,2861 & 24,654 \\
Positive EV & 0,5632 & 10,657 & 0,5686 & 0,4108 & 0,326 & 29,367 \\
p Value & 1,34 & $\mathbf{0 , 0 1}$ & 0,74 & 0,987 & 0,567 & $\mathbf{0 , 0 1}$
\end{tabular}

Adjusted Endometrial Volume Growth Rate

$\begin{array}{lllllll} & \text { GR1 } & \text { GR2 } & \text { GR3 } & \text { GR4 } & \text { GR5 } & \text { Overall GR } \\ \text { Negative AdjEV } & 0,0102 & 0,165 & 0,077 & 0,0063 & 0,005 & 0,0459 \\ \text { Positive Adj EV } & 0,0115 & 0,231 & 0,112 & 0,0083 & 0,007 & 0,061 \\ \text { p Value } & 1,43 & \mathbf{0 , 0 1} & 0,53 & 0,873 & 0,678 & \mathbf{0 , 0 1}\end{array}$

GR1 Growth rate 1 (difference between Basal Moment and Day 6 after controlled ovarian stimulation) GR2 - Growth rate 2 (difference between Day 8 and Day 6 after controlled ovarian stimulation) GR3 - Growth rate 3 (difference between Day 10 and Day 8 after controlled ovarian stimulation) GR4 - Growth rate 4 (difference between Trigger with hCG day and Day 10 after controlled ovarian stimulation) GR5 - Growth rate 5 (difference between Transfer Day and Trigger with hCG day) Overall GR - Overall Growth day (difference between embryo transfer day and Basal moment) Negative EV Negative Group of Endometrial Volume; Positive EV - Positive Group of Endometrial Volume; Negative AdjEV Negative Group of Adjusted Endometrial Volume; Positive AdjEV Positive Group of Adjusted Endometrial Volume. Value of $p<.05$ considered statistically significant through during an ART cycle. The possibility to use non-invasive techniques to determine endometrial receptivity would allow better clinical judgment. This way on the clinical point of view, the decision to transfer a fresh embryo on that same ART cycle or postpone it for a deferred transfer with better endometrial conditions is possible. This non-invasive tool to predict endometrial receptivity will improve clinical setting and allow better understanding of endometrial receptivity. This may be the way to optimize and achieve greater results in ART cycles.

In our study endometrial morphology proven not to be useful and no significant difference was found between the two groups. Also 2-D endometrial thickness showed no difference at early stages of ovarian controlled stimulation, but significant difference could be seen after day 8 of stimulation. These findings are compatible to the ones provided by the literature. The main reason for such may be the fact that subjective tools produce conflicting results and therefore are not able to provide an accurate diagnostic tool for endometrial receptivity assessment.

Endometrial volume and adjusted endometrial volume proven to be more effective with differences shown since early stages of ovarian controlled stimulation. Both groups were similar at baseline but as soon as controlled ovarian stimulation started, the differences between the ones with a positive outcome and the negative group were clearly met.

We have also been able to show differences between the two groups in terms of endometrial and adjusted endometrial volume in early stages of endometrial development under the influence of controlled ovarian stimulation. Higher volumes were seen in the positive controls, but the changes were more evident in early stages (especially between day 6 and day 8 of ovarian 


\section{Endometrial Volume Growth Rate}

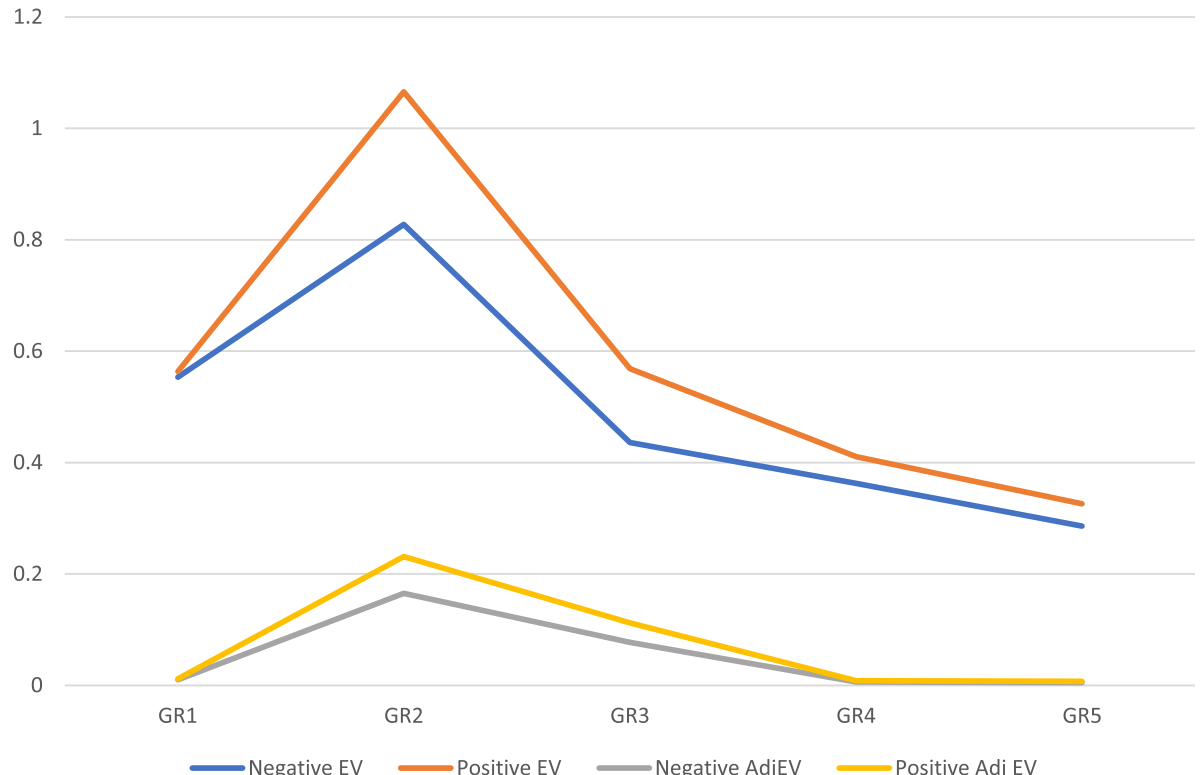

Fig. 4 Endometrial and Adjusted Endometrial Volume Growth Rate (difference between two consecutive measurements). Negative EV - Negative Group of Endometrial Volume; Positive EV - Positive Group of Endometrial Volume; Negative AdjEV - Negative Group of Adjusted Endometrial Volume; Positive AdjEV - Positive Group of Adjusted Endometrial Volume

controlled stimulation). This is also corroborated by the fact that growth rate was statistically higher on the positive group, but the higher difference was met in early stages of endometrial development (between day 6 and day 8). The supraphysiological environment produced by the controlled ovarian stimulation may have leading role in such. Our study showed that serial continuous endometrial volume was significantly higher on the positive group, whereas studies from Kupesic et al., and $\mathrm{Wu}$ et al., [20, 21] have conflicting results in the assessment of endometrial receptivity. The difference can possibly be explained by the fact of serial continuous evaluations better reflect endometrial changes, rather than predetermined single scoop analysis.

The use of a cut off $\geq 5 \mathrm{~mL}$ in endometrial volume in prediction of endometrial receptivity had a good sensitivity and low specificity and may be used as a good test to exclude success. The fact that we can have a tool that indicates a non-optimal endometrium aids in the decision process of postponing embryo transfer for a more suitable and receptive endometrium.

The possibility of real time non-invasive continuous assessment of the endometrium further induces clinicians to better medical decisions. The current study demonstrated that it is possible to evaluate endometrial morphologic parameters, serial endometrial volume and adjusted serial endometrial volume in the coronal plane in accordance with published method by Mercer et al. [22]
All findings may prove to be a useful management tool for clinicians in order to establish a diagnostic tool for better decision making in selective embryo transfers.

Nevertheless, one must always be cautious that artefacts during $3 \mathrm{D}$ analysis may occur due to $2 \mathrm{D}$ imaging process, patient motion during rendering of images and artefacts due to operator choice in the selection of which part of the volume to display [23].

We can highlight as the major strength of this study the awareness to a controversial topic, with a different uptake on the question of non-invasive methods to assess endometrial receptivity. This new methodology of continuous or better yet consecutive serial evaluations ascertain the discriminatory value of volumetric endometrial ultrasound assessment.

Also, the fact of prospective continuous assessment using the same protocol for all subjects can be pointed out as a strength of this study.

The limited number of subjects of our sample can be deemed as a limitation or weakness to this study. Still, this new methodological approach can now be used in a larger setting to provide further information and knowledge to sustain the information already seen.

This study provides a different uptake on the question of non-invasive methods to assess endometrial receptivity. We have successfully been able to obtain limited data concerning a preferential pathway on endometrial volumetry. However larger studies should be carried out to further sustain our findings. 


\section{Conclusions}

Many have been published regarding endometrial receptivity. Still many questions remain without an answer. The possibility to accurately predict implantation is one of the most challenging ones. Progress in embryo transfer and cultures, still relies on the uncertainty of embryo implantation even in the presence of what appears to be a receptive endometrium.

Some techniques have been developed but the results are still controversial, invasive and lacking reliability.

Endometrium is a living, adaptative transformative tissue. Understanding this ability to transform, and the continuity of the process may allow better knowledge of its ability to allow the implantation process of a good quality embryo.

Ultrasonographic advances in the past decades, provides a useful tool to evaluate the morphokynetics of this transformative tissue.

Information of what makes an endometrium receptive may be the key in solving these issues, providing a diagnostic tool that will enhance ART cycles and elective embryo transfers. This will result in more effective transfers and better ART outcomes. Also the possibility to determine in real time endometrial receptivity will shorten the time to birth lapse, thus improving quality of life for infertile couples.

This study showed that endometrial 3D volume analysis as well as adjusted 3D endometrium volume may identify a receptive endometrium as soon as day 6 of ovarian controlled stimulation, and also the results show a high accuracy in detecting the non-receptive endometrium (with a high Predictive Negative Value of 90.1\%). In this way clinicians may be made aware of this possibility, and further enhance its procedures with better knowledge weather or not to perform embryo transfer on that given cycle.

\section{Abbreviations \\ ART : Assisted Reproductive Technology; CHCB: Centro Hospitalar Cova da Beira; ER: Endometrial Receptivity; ERA: Endometrial Receptivity Array; GCP: Good Clinical Practices; hCG: human chorionic gonadotropin; $\mathrm{ICH}$ : International Conference Harmonisation; IU: International Units; ML: Multi-layered; NM: Non Multi-layered; SD : Standard Deviation; WOI: Window of Implantation; 2D: Two Dimensions; 3D : Three Dimensions}

\section{Acknowledgements}

We would like to appraisal M.S. for the help with the statistical analysis of data.

\section{Authors' contributions}

$\mathrm{RSM}, \mathrm{AHO}$ and $\mathrm{JMO}$ are responsible for conception and study design. RSM has been the principal investigator and the principal responsible for acquisition and analysis of data. RSM has been responsible for data interpretation. DVO, AHO and JMO have been responsible for reviewing the article for publication. All authors have read and approved the manuscript, and can be personally accountable for the contributions and can ensure that questions related to the accuracy and integrity of any part of the work.

\section{Funding}

No Grant support on this study.

\section{Availability of data and materials}

Encrypted non-disclosure data available at Open Science Framework database for peer review purpose only. Project name Physical Biomarkers in Endometrial Receptivity with access link: https://osf.io/hr25m/?view_only= 8d5f6dcb8b25420bbd9188382163e7d7

\section{Ethics approval and consent to participate}

The study has been approved by the Ethics Committee of Centro Hospitalar Universitário Cova da Beira (study committee reference number: CHCB 22/ 2017). Oral and written consent was obtained for all willing participants prior to registering for this study. Patient Informed consent to participate in this study CHCB 22/2017.

\section{Consent for publication}

Not Applicable.

\section{Competing interests}

The authors do not report any conflict of interest.

Received: 22 May 2020 Accepted: 28 October 2020

Published online: 03 November 2020

\section{References}

1. Altmae S, Mantinez-Conejero JA, Salumets A, Simon C, Horcajadas JA, Stavreus-Evers A. Endometrial gene expression analysis at the time of embryo implantation in women with unexplained infertility. Mol Hum Reprod. 2010;16:178-87.

2. Navot D, Scott RT, Droesch K, Veeck LL, Liu HC, Rosenwaks Z. The window of embryo transfer and the efficiency of human conception in vitro. Fertil Steril. 1991;55(1):114-8. https://doi.org/10.1016/s0015-0282(16)54069-2.

3. Boomsma CM, Kavelaars A, Eijkemans MJ, Lentjes EG, Fauser BC, Heijnen CJ, Macklom NS. Endometrial secretion analysis identifies a cytokine profile predictive of pregnancy in IVF. Hum Reprod. 2009;24:1427-35.

4. Bourgain C, Devroey P. The endometrium in stimulated cycles for IVF. Hum Reprod Update. 2003;9:515-22.

5. Casado-Vela J, Rodriguez-Suarez E, lloro I, Ametzazurra A, Alkorta N, GarciaVelasco JA, Matorras R, Prieto B, Gonzales S, NAgore D, Simon L, Elortza F. Comprehensive proteomic analysis of human endometrial fluid aspirate. J ProteomeRes. 2009;8:4622-32.

6. Ellington AA, Kullo IJ, Bailey KR, Klee GG. Measurement and quality control issues in multiplex protein assays: a case study. Clin Chem. 2009:55:1092-9.

7. Fatemi HM, Popovic. Teodorovic, B. Implantation in assisted reproduction: a look at endometrial receptivity. Reprod Biomed Online. 2013;27:530-8.

8. Labarta E, Mariani G, Holtman N, Celada P, Remohí J, Bosch E. 2017. Low serum progesterone on the day of embryo transfer is associated with a diminished ongoing pregnancy rate in oocyte donation cycles after artificial endometrial preparation: a prospective study. Hum Reprod. 2017:32(12): 2437-42.

9. Hannan NJ, Stephens AN, Rainczuk A, Hincks C, Rombauts $\sqcup$, Salamonsen LA. 2D-DiGE analysis of the human endometrial secretome reveals differences between receptive and nonreceptive states in fertile and infertile women. J Proteome Res. 2010;9:6256-64.

10. Haouzi D, Dechaud H, Assou S, De Vos J, Hamamah S. Insights into human endometrial receptivity from transcriptomic and proteomic data. Reprod BioMed Online. 2012;24:23-34.

11. Silva Martins R, Helio Oliani A, Vaz Oliani D, Martinez de Oliveira J. Subendometrial resistence and pulsatility index assessment of endometrial receptivity in assisted reproductive technology cycles. Reprod Biol Endocrinol. 2019;17(1):62. https://doi.org/10.1186/s12958-019-0507-6.

12. Hou Z, Zhang Q, Zhao J, Xu A, He A, Huang X, Xie S, Fu J, Xiao L, Li Y. Value of endometrial echo pattern transformation after hCG trigger in predicting IVF pregnancy outcome: a prospective cohort study. Reprod Biol Endocrinol. 2019;17:74. https://doi.org/10.1186/s12958-019-0516-5.

13. Lessey BA. Assessment of endometrial receptivity. Fertil Steril. 2011;96:522-9

14. Lessey BA. The pathologists are free to go, or are they? Fertile Steril. 2013; 99:350-1.

15. Sher G, Dodge S, Maassarani G, Knutzen V, Zouves C, Feinman M. 1993. Management of suboptimal sonographic endometrial patterns in patients undergoing in-vitro fertilization and embryo transfer. Hum Reprod. 1993 Mar;8(3):347-9. 
16. Mains $L$, Van Voohirs BJ. Optimizing the technique of embryo transfer. Fertile Steril. 2010;94:785-90.

17. Navot D, Bergh PA, Williams MA, GArrisi GJ, Guzman I, Sandler B, Grunfeld L. Poor oocyte quality rather than implantation failure as a cause of agerelated decline in female fertility. Lancet. 1991;337:1375-7.

18. Ruiz-Alonso M, Blesa D, Simon C. The genomics of the human endometrium. Biochim Biophys Acta. 2012;1822:1931-42.

19. Thelen JJ, Miernyk JA. The proteomic future: where mass spectroscopy should be taking us. Biochem J. 2012;444:169-81.

20. Kupesi S, Bekavac I, Bjelos D, Kurjak A. Assessment of endometrial receptivity by transvaginal color Doppler and 3Dpower Doppler ultrasonography in patients undergoing in vitro fertilization procedures. J Ultrasound Med. 2001;20:125-34

21. Wu HM, Chiang CH, Huang HY, Chao AS, Wang HS, Soong YK. Detection of the subendometrial vascularization flow index by 3D ultrasound may be useful for predicting the pregnancy rate for patients undergoing in vitro fertilization-embryo transfer. Fertil Steril. 2003;79:507-11.

22. Merce LT, Barco MJ, Bau S, Tryoano J. Are endometrial parameters by $3 D$ ultrasound and power Doppler angiography related to in vitro fertilization/ embryo transfer outcome? Steril Fertil. 2008;89:112-7.

23. Vilella F, Ramirez LB, Simon C. Lipidomics as an emerging tool to predict endometrial receptivity. Fertile Steril. 2013;99:1100-6.

\section{Publisher's Note}

Springer Nature remains neutral with regard to jurisdictional claims in published maps and institutional affiliations.

Ready to submit your research? Choose BMC and benefit from:

- fast, convenient online submission

- thorough peer review by experienced researchers in your field

- rapid publication on acceptance

- support for research data, including large and complex data types

- gold Open Access which fosters wider collaboration and increased citations

- maximum visibility for your research: over $100 \mathrm{M}$ website views per year

At BMC, research is always in progress.

Learn more biomedcentral.com/submissions 